\title{
Analysis of the Transient Dynamics of Microwave Oscillators
}

\author{
Sergio Sancho, Member, IEEE, Almudena Suárez, Fellow, IEEE, Franco Ramírez, Senior Member, \\ IEEE, and Mabel Pontón, Member, IEEE
}

\begin{abstract}
A semi-analytical method for the global prediction and understanding of the transient dynamics of oscillator circuits is presented. It covers both the linear and nonlinear transient stages, which are related with the circuit generalized eigenvalues, here introduced for the first time. The transient model relies on the application of the implicit-function theorem to the harmonicbalance system, in order to derive a reduced-order nonlinear differential equation from a given observation node. This requires the extraction of a nonlinear admittance function, depending on the voltage excitation and oscillation frequency, which is done with a forcing auxiliary generator. The linearization of this admittance function for each excitation amplitude provides a sequence of linear ordinary differential equations, describing the system dynamics in the vicinity of each point of the transient trajectory, which can be reconstructed from the expression of the solution increment at each time step. The sequence of differential equations provides a set of generalized eigenvalues, responsible for the acceleration or deceleration of the oscillation growth and capable to detect spurious transient frequencies. The concept of escape time, or time required by the transient trajectory to go through a certain interval of amplitude values, is also introduced, for the first time to our knowledge. The method has been successfully applied to analyze the transient dynamics of several FET oscillators, including dual-frequency oscillators and switched oscillators.
\end{abstract}

Index Terms-Frequency-domain analysis, microwave oscillator, transient analysis.

\section{INTRODUCTION}

$\mathrm{T}_{\mathrm{b}}$ transient dynamics is a key characteristic of the oscillator behavior [1]-[7] that highly influences the performance of the system in which it is embedded. For example, in the case of mobile communication devices, where the system is powered up and down, the time required for the oscillator to achieve the steady state affects the energy consumed. Moreover, anomalous switching transients can temporarily stress the system and/or cause inference. Time-domain integration is the natural choice to predict the oscillator transient. However, in the microwave range, numerical difficulties are often encountered, due to the common presence of distributed elements and the long transient duration in comparison with the solution period. On the other hand, the envelope-transient approach [8]-[12] relies on a Fourier-series representation of the circuit variables with timevarying harmonic terms, which enables the derivation of a nonlinear differential equation system in these slowly varying terms. This system is integrated at a significantly larger time scale than the one required for the standard time-domain analysis, which enables an efficient simulation. Furthermore, the modeling of the distributed elements is limited to a certain bandwidth about the harmonic components, which is less demanding from a numerical viewpoint.

At system level, compact oscillator models are used for the analysis of coupled oscillator systems [13],[14], phase-locked loops and self-injection locked circuits, for instance. The oscillators are usually described with Van der Pol models [15][18], which, in most cases, cannot accurately predict the behavior of transistor-based circuits. To address this problem, a reduced-order model, extracted from circuit-level harmonicbalance (HB) simulations, was proposed in [13],[14]. The model is obtained by linearizing an outer-tier admittance function $Y$, calculated at a given observation node, about the free-running periodic solution. More specifically, it is given by the amplitude and frequency derivatives of this function, calculated through finite differences [13],[14]. As a result, its prediction capabilities are limited to small transient perturbations about the steady state.

To extend the numerical model in order to account for the build-up transient, the preliminary work [19], [20] presents the derivation of a first-order envelope-domain equation from a single observation node. The model relies on a full extraction of an outer-tier admittance function $Y(V, \omega)$ from $\mathrm{HB}$, which depends on the excitation amplitude $V$ and frequency $\omega$. The first-order envelope domain equation is obtained by considering a time-varying amplitude $V(t)$ and applying a first-order Taylor series expansion of $Y(V, \omega)$ in $j \omega$, for each $V$. Then, the multiplication by $j \omega$ gives rise to a time differentiation of the node voltage, in a manner similar to [21], which leads to a firstorder nonlinear differential equation.

As stated, the numerical function $Y(V, \omega)$ enables a general procedure for a realistic prediction of the oscillator behavior. However, the first-order assumption [19], [20] will fail if the transient effects cannot be accurately described through a firstorder Taylor series expansion in $j \omega$. To cope with this limitation, this work considers a general dependence on $j \omega$. This involves a significant challenge in the resolution of the nonlinear differential equation because there is no explicit frequency dependence that can be easily associated with time differentiations. Two different ways to integrate the equation will be considered: a local linearization (LL) method [22]-[29] and a nonlinear iterative procedure [30]. 
The LL method is based on the discretization of the time variable in small intervals $\left(t_{n}, t_{n+1}\right)$, in which the original nonlinear differential equation is approached by a linear one, obtained by linearizing the admittance function about the amplitude $V_{n}$ at $t_{n}$. As in all the LL methods [22]-[29], this resolution is exponential, in the sense that, in each time interval $\left(t_{n}, t_{n+1}\right)$, the transient depends on the eigenvalues of the system Jacobian matrix, evaluated at $t_{n}$. Here, these eigenvalues are calculated in an indirect manner, applying, under $V$ variations, a series of pole-zero identification to a frequency-domain function, derived from the characteristic system. It is taken into account that all the transfer functions that can be defined in a linearized system share the same denominator [31]-[40], so the exponentials are unique up to the accuracy limits of the polezero identification.

The linearization methods are approximate and have an error proportional to the square of the time step [22]-[29]. However, the decomposition into exponential terms enables the detection and monitoring of spurious frequencies. In fact, the generalized eigenvalues evolve during the transient and are responsible for the onset and extinction of spurious oscillations during this transient stage. They also govern acceleration or deceleration of the oscillation growth. Thus, the new method will provide insight into the causes for irregular transient responses. The transient prediction can be refined using an iterative integration method, such as the backward Euler rule [30].

The paper is organized as follows. Section II describes the time-frequency formulation, including the calculation of the nonlinear admittance function. Section III presents the narrowband analysis, extended with the calculation of a newly defined escape time. Section IV describes the wideband analysis, including the two different ways to solve the implicit envelope-domain equation. Section $\mathrm{V}$ presents various applications of the new model, with experimental measurements, including a dual-frequency oscillator and a switched oscillator.

\section{TIME-FREQUENCY FORMULATION}

Let a free-running oscillator be analyzed with the HB method be considered. The HB system is expressed in terms of its error vector as $\bar{H}(\bar{X}, \omega)=0$, where $\omega$ is the unknown fundamental frequency and the phase of one of the state variables in $\bar{X}$ has been arbitrarily set to zero, due to the autonomy of the freerunning oscillator solution. The total current is equal to zero at all the circuit nodes, so, when considering a particular observation node $q$, one can write the following complex equation at the fundamental frequency:

$$
I_{1}\left(V, \phi, \omega, \bar{X}^{\prime}\right)=0
$$

where $I_{1}$ is the fundamental-frequency component of the total current entering the node, $V$ and $\phi$ are the amplitude and phase of the first harmonic of the voltage $v(t)$ at the observation node and $\bar{X}^{\prime}$ is the vector containing the rest of the
HB-system unknowns. Defining $\bar{H}^{\prime}\left(V, \phi, \omega, \bar{X}^{\prime}\right)=0$ as the subsystem composed by the remaining $\mathrm{HB}$ equations, the circuit is governed by:

$$
\begin{aligned}
& \bar{H}^{\prime}\left(V, \phi, \omega, \bar{X}^{\prime}\right)=0 \\
& I_{1}\left(V, \phi, \omega, \bar{X}^{\prime}\right)=0
\end{aligned}
$$

The Implicit-Function Theorem [41] states that, if the Jacobian matrix of subsystem (2)(a) is not singular, it is possible to express $\bar{X}^{\prime}$ in terms of $(V, \omega)$, obtaining [42]:

$$
\begin{aligned}
& I_{1}\left[V, \phi, \omega, \bar{X}^{\prime}(V, \phi, \omega)\right]=Y(V, \omega) X_{1} \\
& X_{1} \equiv V e^{j \phi}
\end{aligned}
$$

where $Y(V, \omega)$ is the first-harmonic admittance function at the observation node. To ease the reading the most significant symbols and quantities used in the theoretical analysis are presented in Table I.

Table I

\begin{tabular}{|l|l|}
\hline Symbol & Description \\
\hline$V, \phi$ & First harmonic amplitude and phase \\
\hline$g(V)$ & Growth-rate function \\
\hline$e(V)$ & Escape time density function \\
\hline$Y(V, \omega)$ & $\begin{array}{l}\text { Admittance function evaluated at the } \\
\text { first harmonic component of the two-tier } \\
\text { system (3) }\end{array}$ \\
\hline$\lambda_{1}(V), \ldots, \lambda_{p}(V)$ & Generalized eigenvalues \\
\hline$F_{V}^{+}(V, s)$ & $\begin{array}{l}\text { Laplace-domain function whose poles } \\
\text { agree with the generalized eigenvalues }\end{array}$ \\
\hline
\end{tabular}

\section{A. Extraction of the nonlinear admittance function}

The nonlinear admittance function $Y(V, \omega)$ is extracted in the following manner. First, an auxiliary generator in series with an ideal band-pass filter, exhibiting the admittance $Y_{f}(\omega)=\delta\left(\omega-\omega_{A G}\right)$, is connected in parallel at the observation node $q$, as shown in Fig. 1(a). Then, a double sweep in the variables $(\omega, V)$ is performed, obtaining the admittance function $Y(V, \omega)$ as:

$$
Y(V, \omega)=I_{1}\left(V_{A G}, \omega_{A G}\right) / V_{A G}, \quad V_{A G}=V, \omega_{A G}=\omega
$$

where the first harmonic current $I_{1}\left(V_{A G}, \omega_{A G}\right)$ is calculated through circuit-level HB simulation of the oscillator, after setting the amplitude and frequency of the AG to $V_{A G}=V, \omega_{A G}=\omega$. Due to the oscillator autonomy, the AG phase $\phi_{A G}$ can be arbitrarily set to zero. The amplitude $V$ must be swept in the range of amplitude values expected to be covered by the transient trajectory. 

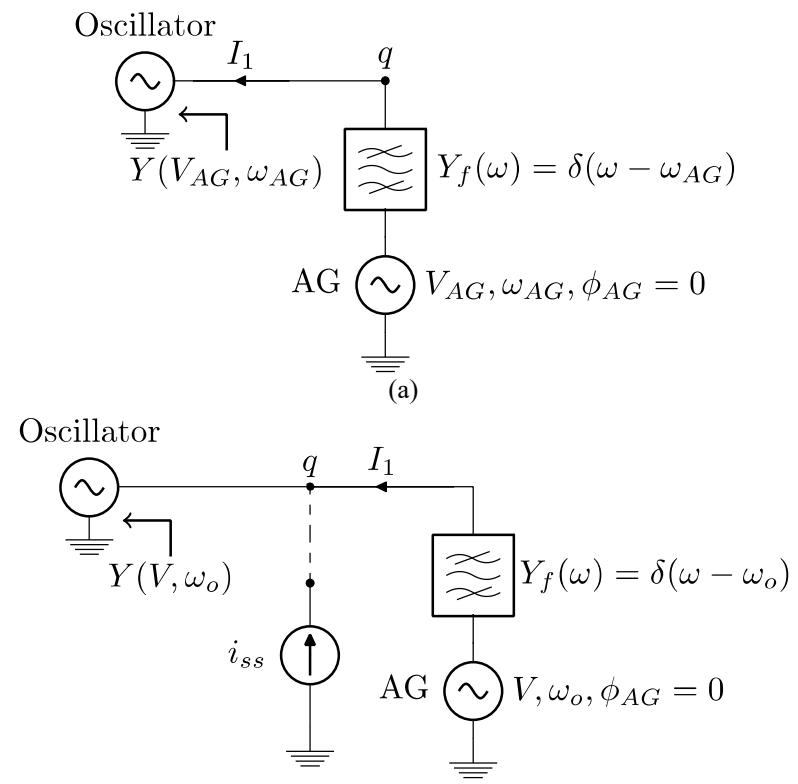

(b)

Fig. 1. Schematic of the auxiliary generator (AG) connected to an observation node $q$ of the oscillator circuit. (a) Use of the AG to calculate the admittance function $Y(V, \omega)$. (b) Inclusion of a small signal current source for pole-zero identification at circuit level.

\section{B. Envelope-domain equation}

During the transient state, the harmonic components of the voltage signal $v(t)$ are time-varying: $v(t)=\sum V_{n}(t) e^{j n \omega_{o} t}$, where $\omega_{o}$ is the steady state-oscillation frequency. As a result the outer-tier function $Y$ must include the action of a time differentiator [21]. Note that the circuit is not in steady state, which, in the frequency domain, gives rise to a complex frequency, which, in the envelope domain, is expressed as $\omega_{o}+s / j$, where $\omega_{o}$. Multiplication by $s$ of the time-varying terms is equivalent to a time differentiation [21], in consistency with the time differentiation associated with a multiplication by $s$ in the Laplace domain. Thus, departing from equation (3), one obtains:

$$
Y\left[V(t), \omega_{o}+D_{t} / j\right] X_{1}(t)=0
$$

where $D_{t}$ is the time-derivative operator. Equation (5) enables the derivation of a reduced-order model, able to account for build-up transients. In the following, the two cases of narrowband and wideband envelopes will be distinguished.

\section{NARROWBAND TRANSIENT}

In previous works [19], [20] the time-varying harmonic component $X_{1}(t)$ was treated as a narrow-band signal, assuming that its high-order time derivatives were negligible. Under this assumption, (5) can be expressed as:

$$
\begin{aligned}
& Y\left(V, \omega_{o}\right) X_{1}+\frac{\partial Y\left(V, \omega_{o}\right)}{\partial \omega} \frac{D_{t}}{j}\left(X_{1}\right)= \\
& Y\left(V, \omega_{o}\right) X_{1}+\frac{\partial Y\left(V, \omega_{o}\right)}{\partial(j \omega)} \dot{X}_{1}=0
\end{aligned}
$$

For the sake of compactness, the following change of notation will be performed:

$$
\begin{aligned}
& a_{0}(V)=Y\left(V, \omega_{o}\right) \\
& a_{1}(V)=\frac{\partial Y\left(V, \omega_{o}\right)}{\partial j \omega}
\end{aligned}
$$

Both $a_{0}$ and $a_{1}$ can be obtained from the numerical function $Y(V, \omega)$ in a straightforward manner. From (6), one can extract the equations governing the amplitude and phase dynamics:

$$
\begin{gathered}
\dot{V}=\frac{\operatorname{Re}\left[a_{0}(V)\right] \operatorname{Re}\left[a_{1}(V)\right]+\operatorname{Im}\left[a_{0}(V)\right] \operatorname{Im}\left[a_{1}(V)\right]}{\left|a_{1}(V)\right|^{2}} \equiv g(V) \\
\dot{\phi}=\frac{\operatorname{Re}\left[a_{0}(V)\right] \operatorname{Im}\left[a_{1}(V)\right]-\operatorname{Im}\left[a_{0}(V)\right] \operatorname{Re}\left[a_{1}(V)\right]}{\left|a_{1}(V)\right|^{2}}
\end{gathered}
$$

where $\operatorname{Re}()$ and $\operatorname{Im}()$ indicate real and imaginary parts, respectively. Note that the instantaneous oscillation frequency is $\omega_{o}+\dot{\phi}(t)$. Due to the oscillator autonomy, the system is invariant to any constant phase shift. This is because the time evolution of system (8)-(9) is determined by the single state variable $V$. The nonlinear function $g(V)$ is a growth-rate function that governs the amplitude dynamics.

In steady-state conditions, $\dot{X}_{1}=0$ in (6), which is fulfilled by the steady-state values $V=0, V_{o}$ that make $a_{0}(V)=0$. For these values, which correspond to the dc solution and the steady-state oscillation, equations (8)-(9) predict that both the amplitude and phase variables fulfill $\dot{V}=\dot{\phi}=0$.

In narrowband conditions, the function $g(V)$ will contain the information on the stability of the steady-state solution $V_{s s}$. The amplitude behavior in the vicinity of $V_{s s}$ can be evaluated by expressing the first harmonic amplitude as $V(t)=V_{s s}+\Delta V(t)$. Introducing this expression in the ordinary differential equation (ODE) [27] (8), the time evolution of the amplitude deviation is given by:

$$
\Delta \dot{V}(t) \cong g\left(V_{s s}\right)+g^{\prime}\left(V_{s s}\right) \Delta V(t)=g^{\prime}\left(V_{s s}\right) \Delta V(t)
$$

where it has been taken into account that $g\left(V_{s s}\right)=0$. According to equation (10), for $g^{\prime}\left(V_{s s}\right)<0$ (in the vicinity of the steadystate solution), the amplitude deviation decreases with time and the system trajectories are attracted by $V_{s s}$. In this case, the solution $V_{s s}$ is stable whereas. For $g^{\prime}\left(V_{s s}\right)>0$, the amplitude deviation increases with time and $V_{s s}$ is unstable.

\section{A. Application to a FET-based oscillator}

The analysis based on (8) will be illustrated by its application to the FET-based oscillator at $5 \mathrm{GHz}$ of Fig. 2(a). The drain bias voltage is $V_{D D}=1 \mathrm{~V}$ and the varactor diode has been set to 
$V_{d}=1 \mathrm{~V}$. The observation node $q$ has been placed at the gate port. In Fig. 2(b), the real and imaginary parts of the function $Y(V, \omega)$ have been represented versus the voltage amplitude $V$, which has been varied in the range $[0,1] \mathrm{V}$. The representation has been carried out for the frequency values $f=\omega / 2 \pi=f_{o}=5 \mathrm{GHz}$ and $f=f_{o}+40 \mathrm{MHz}$. As can be seen, for $f=f_{o}$ the admittance function vanishes at the steadystate amplitude $V_{o}=0.59 \mathrm{~V}$.

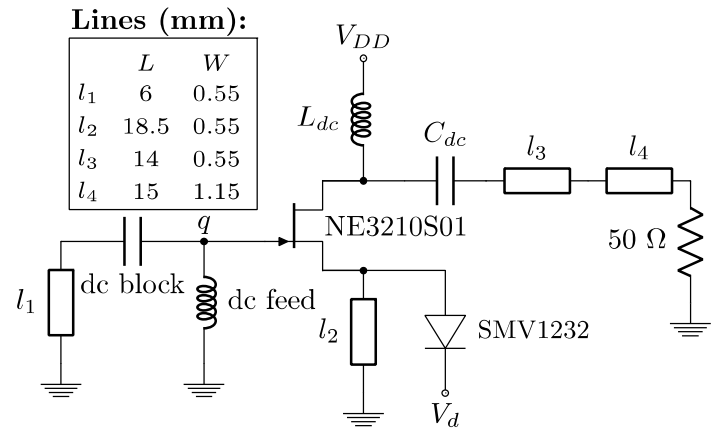

(a)

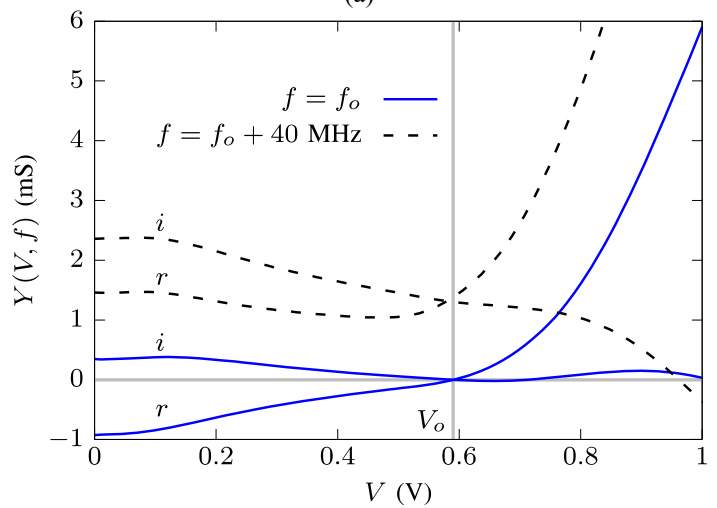

(b)

Fig. 2. Application of the first-order technique. (a) Schematic of the FET-based oscillator at $5 \mathrm{GHz}$, where the variables $L$ and $W$ are the length and width of the circuit transmission lines. The time delay of each line is $\tau_{1}=32 \mathrm{ps}$, for $L_{1}$, $\tau_{2}=110 \mathrm{ps}$, for $L_{2}, \tau_{3}=76 \mathrm{ps}$, for $L_{3}$, and $\tau_{4}=84 \mathrm{ps}$, for $L_{4}$. (b) Calculation of the components $a_{o}(V)=Y\left(V, \omega_{0}\right)$ and $a_{1}(V)$. The real and imaginary parts of $Y(V, \omega)$ (denoted by $r, i$ ) have been represented versus $V$ for $f=f_{o}$ and $f=f_{o}+40 \mathrm{MHz}$, where $f_{o}=5 \mathrm{GHz}$. For each amplitude $V$, the function $a_{1}(V)$ in $(7)$ is obtained from the numerical frequency derivative of $Y(V, \omega)$, calculated at $\omega=\omega_{0}=2 \pi f_{o}$.

In Fig. 3(a), the function $g(V)$ has been represented for two different values of the varactor diode bias $V_{d}=1 \mathrm{~V}$ and $V_{d}=1.08 \mathrm{~V}$. Note that, since $\dot{V}=g(V)$, this representation provides the system trajectory in the phase space $(V, \dot{V})$. As can be observed, $g(V)$ vanishes at the steady state values $V=0, V_{o}\left(V_{d}\right)$, corresponding to the dc solution and steady-state oscillation. According to the derivative $g^{\prime}(V)$, the dc solution is unstable and the steady-state oscillation is stable. In the transient between both solutions the amplitude grows faster for $V_{d}=1 \mathrm{~V}$, since $g(V)$ is bigger. In the same figure, the trajectories are validated through a comparison with circuitlevel envelope-transient simulations (and with the general nonlinear integration method presented in Section IV.D). In the circuit-level envelope-transient, to avoid the convergence to the coexisting unstable dc solution, the simulation has been carried out with the aid of an $\mathrm{AG}$, connected to the circuit at the initial time only, as described in [43].
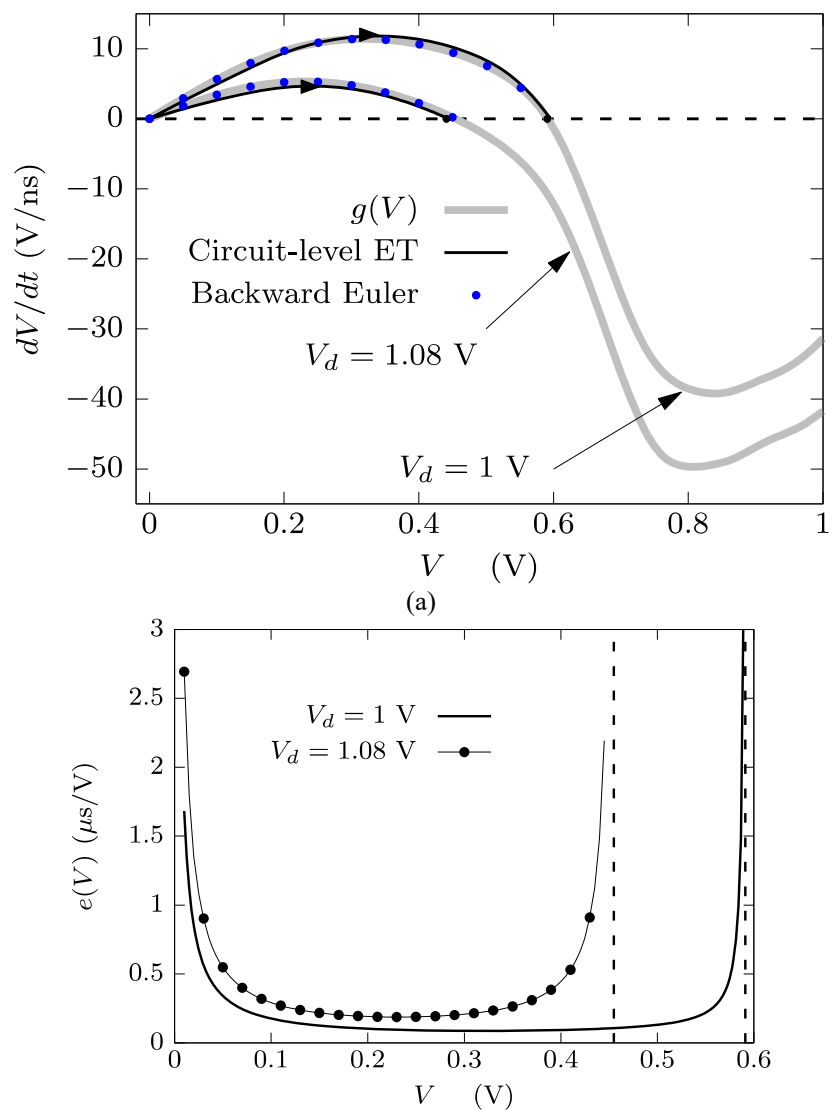

(b)

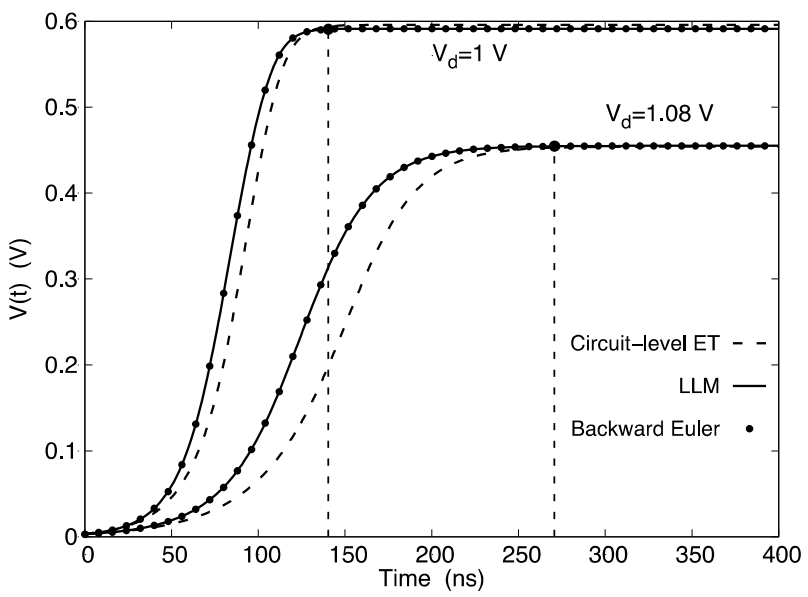

(c)

Fig. 3. Application of the first-order technique. (a) Growth-rate function $g(V)$ for two values of the varactor bias $V_{d}$. The amplitude grows faster for $V_{d}=1 \mathrm{~V}$ , since $g(V)$ is bigger. The results have been validated with circuit-level envelope-transient (ET) simulations and with the nonlinear integration method in Section IV.D. (b) Escape-time density for two values of the varactor bias $V_{d}$. The vertical dashed lines indicate, in each case, the steady-state oscillation amplitude. (c) Circuit-level envelope transient simulations of the transient trajectory. The trajectories obtained through the LL and backward-Euler methods have been superimposed. The vertical lines indicate the estimated time to reach the $99.9 \%$ of the steady state amplitude value in each case. 


\section{B. Escape time}

Once the growth-rate function $g(V)$ defined in (8) has been calculated, the transient dynamics of the oscillator amplitude, from any initial condition $V=V_{I C}$ to the steady state $V=V_{o}$, is fully determined by the first-order ODE (8). The time required to reach a given amplitude value $V$ from an initial condition $V_{I C}$ can be obtained from (8) as:

$$
t=\int_{V_{I C}}^{V} e(V) d V=f\left(V, V_{I C}\right), \quad e(V)=\frac{1}{g(V)}
$$

where the function $e(V)$ is the escape-time density, since $e(V) d V$ provides the time required to go through the interval $[V, V+d V]$. The growth-rate function $g(V)$ vanishes when evaluated at any steady-state amplitude $V_{s s}$. This is because, at the steady states, equation (3) is fulfilled, which implies $a_{0}\left(V_{s s}\right)=Y\left(V_{s s}, \omega_{o}\right)=0$. Thus, at the steady-state solutions, the escape time density $e(V)$ becomes infinite, since they are fixed points.

In Fig. 3(b), the escape-time density $e(V)$ has been represented for two values of the varactor bias $V_{d}$. The density is smaller for $V_{d}=1 \mathrm{~V}$, which implies that the oscillator response is faster. As seen in the figure, $e(V) \rightarrow \infty$ at the dc and oscillatory steadystate solutions.

Using the escape-time density $e(V)$, one can obtain, for instance, the time required to reach a $99.9 \%$ of the amplitude of the stable free-running periodic steady state. It is also possible to analyze the impact of a given circuit parameter on the transient duration. In the case of the oscillator in Fig. 2(a), starting from $V_{I C}=3 \mathrm{mV}$, the time required to reach a $99.9 \%$ of the steady-state amplitude is $t=140 \mathrm{~ns}$ for $V_{d}=1 \mathrm{~V}$, and $t=270$ $\mathrm{ns}$, for $V_{d}=1.08 \mathrm{~V}$. These results are validated with circuit-level envelope-transient simulations in Fig. 3(c).

\section{WIDEBAND ANALYSIS OF THE OSCILLATOR TRANSIENT}

There are oscillators whose admittance function $Y(V, \omega)$ exhibits a complex dependence on $\omega$, which cannot be accurately represented with a first-order Taylor series expansion in the frequency $\omega$. To address this situation, the general equation (5) will be considered, which exhibits an implicit dependence on both the amplitude $V$ and frequency $\omega$. In the following, it will be solved in two different ways: through a local linearization method and through a nonlinear integration method, based on an iterative procedure.

\section{A. Local linearization method}

To solve (5) through a local-linearization method, the time axis will be divided into subintervals, performing a linearization of the admittance function $Y$ with respect to $V$ at the initial time point of each subinterval.

Let $(V, \phi)$ be the first harmonic amplitude and phase values corresponding to a given time point of the transient trajectory.
After a sufficiently small time $t$, the amplitude and phase can be expressed as $V+\Delta V(t)$ and $\phi+\Delta \phi(t)$, respectively, where $\Delta V(t)$ and $\Delta \phi(t)$ are small quantities. Linearizing $Y\left[V(t), \omega_{o}+D_{t} / j\right]$ in (5) about $V$, while preserving the global dependence on $\omega_{o}+D_{t} / j$, one obtains:

$$
\begin{aligned}
& Y\left[V(t), \omega_{o}+D_{t} / j\right] \cong \\
& Y\left(V, \omega_{o}+D_{t} / j\right)+\frac{\partial Y\left(V, \omega_{o}+D_{t} / j\right)}{\partial V} \Delta V
\end{aligned}
$$

Then, introducing (12) into (5), one obtains:

$Y\left(V, \omega_{o}+D_{t} / j\right) X_{1}(t)+\frac{\partial Y\left(V, \omega_{o}+D_{t} / j\right)}{\partial V} \Delta V(t) X_{1}(t)=0$

Equation (13) is composed by two terms, which will be analyzed individually. The first term can be expressed as:

$$
\begin{aligned}
& Y\left(V, \omega_{o}+D_{t} / j\right) X_{1}(t)= \\
& =\left[Y\left(V, \omega_{o}+D_{t} / j\right)-Y\left(V, \omega_{o}\right)\right] X_{1}(t)+Y\left(V, \omega_{o}\right) X_{1}(t)= \\
& =e^{j(\phi+\Delta \phi)}\left[Y\left(V, \omega_{o}+D_{t} / j\right)-Y\left(V, \omega_{o}\right)\right](\Delta V+j V \Delta \phi)+ \\
& +Y\left(V, \omega_{o}\right) X_{1}(t)
\end{aligned}
$$

where the time-derivative operator $D_{t}$ has been applied neglecting high-order terms in the perturbation variables:

$$
\frac{d^{k} X_{1}(t)}{d t^{k}} \approx e^{j(\phi+\Delta \phi)} \frac{d^{k}}{d t^{k}}(\Delta V+j V \Delta \phi), \quad k>0
$$

The second term in (13) can be expressed as:

$$
\begin{aligned}
& \frac{\partial Y\left(V, \omega_{o}+D_{t} / j\right)}{\partial V} \Delta V(t) X_{1}(t)= \\
& =e^{j(\phi+\Delta \phi)} \frac{\partial Y\left(V, \omega_{o}+D_{t} / j\right)}{\partial V} \Delta V
\end{aligned}
$$

where, as in the previous case, $D_{t}$ has been applied neglecting high-order terms:

$$
\frac{d^{k} X_{1}(t) \Delta V(t)}{d t^{k}} \approx e^{j(\phi+\Delta \phi)} V \frac{d^{k} \Delta V}{d t^{k}}, \quad k \geq 0
$$

Now, introducing (14) and (16) in (13), one obtains:

$$
\begin{aligned}
& {\left[Y\left(V, \omega_{o}+D_{t} / j\right)+\frac{\partial Y\left(V, \omega_{o}+D_{t} / j\right)}{\partial V}\right] \Delta V+} \\
& +\left[Y\left(V, \omega_{o}+D_{t} / j\right)-Y\left(V, \omega_{o}\right)\right] j V \Delta \phi+Y\left(V, \omega_{o}\right) V=0
\end{aligned}
$$

For each amplitude $V$, equation (18) is a complex linear ODE in the real variables $(\Delta V(t), \Delta \phi(t))$. To complete the system, the same procedure will be followed for the harmonic $X_{-1}(t)$ corresponding to the frequency $\omega=-\omega_{o}$. This provides:

$$
\begin{aligned}
& Y\left(V, \omega_{o}+D_{t} / j\right) X_{-1}(t)+ \\
& +\frac{\partial Y\left(V,-\omega_{o}+D_{t} / j\right)}{\partial V} \Delta V(t) X_{-1}(t)=0
\end{aligned}
$$


which, following the same procedure as in the previous case, leads to:

$$
\begin{aligned}
& {\left[Y\left(V,-\omega_{o}+D_{t} / j\right)+\frac{\partial Y\left(V,-\omega_{o}+D_{t} / j\right)}{\partial V}\right] \Delta V-} \\
& -\left[Y\left(V,-\omega_{o}+D_{t} / j\right)-Y\left(V,-\omega_{o}\right)\right] j V \Delta \phi+Y\left(V, \omega_{o}\right) V=0
\end{aligned}
$$

Equations (18) and (20) constitute a linear time-invariant (LTI) system providing the time evolution of the perturbation components $(\Delta V(t), \Delta \phi(t))$. Note that this system is nonhomogeneous during the transient state but becomes homogeneous, as expected, at the steady-state oscillation, since $Y\left(V_{o}, \omega_{o}\right)=0$. The system (18)-(20) can be solved by applying the theory of linear ODEs [44], so, at each stage, the amplitude increment $\Delta V(t)$ can be expressed as:

$$
\Delta V(t)=\sum_{i=1}^{p} C_{i}\left(e^{\lambda_{i}(V) t}-1\right)
$$

where $p$ is the number of generalized eigenvalues and the constants $\left\{C_{i}\right\}_{i=1}^{p}$ are determined by the initial conditions on the state variables. Because $Y$ depends implicitly in $\omega$ it is not possible to know a priori the order $p$. This order will empirically determined through pole-zero identification [31] [40], as described next.

\section{B. Eigenvalue calculation}

In the following, we will demonstrate that the exponential terms in (21) can be easily obtained through a pole-zero identification procedure. The rigorous demonstration has some complexity, but once the validity of the procedure has been proved, its practical application by the user is straightforward.

For the derivation of the set of generalized eigenvalues $\rho(V)=\left\{\lambda_{1}(V), \ldots, \lambda_{p}(V)\right\}, \quad$ a solution of the form $\Delta V(t)=A_{V} e^{s t}$ and $\Delta \phi(t)=A_{\phi} e^{s t}$ is introduced in (18)-(20). Eliminating the exponential $e^{s t}$ and assembling the terms effecting $A_{V}$ and $A_{\phi}$, one obtains the following characteristic system:

$$
\begin{aligned}
A(V, s)\left(\begin{array}{c}
A_{V} \\
A_{\phi}
\end{array}\right) & =\overline{0}, \\
A(V, s) & \equiv\left(\begin{array}{ll}
b_{V}^{+}(V, s) & b_{\phi}^{+}(V, s) \\
b_{V}^{-}(V, s) & b_{\phi}^{-}(V, s)
\end{array}\right)
\end{aligned}
$$

where:

$$
\begin{aligned}
& b_{V}^{ \pm}(V, s) \equiv Y\left(V, \pm \omega_{o}+s / j\right)+V \frac{\partial Y\left(V, \pm \omega_{o}+s / j\right)}{\partial V}, \\
& b_{\phi}^{ \pm}(V, s) \equiv \pm j V\left[Y\left(V, \pm \omega_{o}+s / j\right)-Y\left(V, \pm \omega_{o}\right)\right]
\end{aligned}
$$

The subscript $+(-)$ indicates that the function is evaluated at $\omega_{o}\left(-\omega_{o}\right)$. The subscript $V(\phi)$ indicates that the function affects $A_{V}\left(A_{\phi}\right)$. At each integration stage, the generalized eigenvalues are the roots of the characteristic determinant det $A(V, s)$. Since the matrix $A(V, s)$ does not depend on $\phi$, the set $\rho(V)$ only depends on the amplitude $V$ of the point under evaluation. As will be shown, this is a relevant property for the practical calculation of the exponential terms in (21). The matrix $A(V, s)$ fulfills $A(V, 0) \bar{u}_{\phi}=\overline{0}, \forall V$, with $\bar{u}_{\phi}=[0,1]^{t}$, so $\operatorname{det} A(V, s)$ has a root $s=0$. This is consistent with the oscillator autonomy, and implies that the transient trajectory remains invariant under any constant shift in the phase variable $\phi$. Note that the same property was obtained in the narrowband case of Section III. From a numerical viewpoint, and because the components of $A(V, s)$ are numerically but not analytically known, better accuracy is obtained through the pole-zero identification of the components of the inverse matrix:

$$
A(V, s)^{-1} \equiv\left(\begin{array}{ll}
F_{V}^{+}(V, s) & F_{V}^{-}(V, s) \\
F_{\phi}^{+}(V, s) & F_{\phi}^{-}(V, s)
\end{array}\right)
$$

The set $\rho(V)$ of generalized eigenvalues can be obtained through the pole-zero identification [31]-[40] of $F_{V}^{+}(V, s)$, evaluated at $s=j \Omega$. The poles of this function are the roots of the characteristic determinant det $A(V, s)$, except the roots $s=0$. Note that the function $F_{V}^{+}(V, j \Omega)$ is derived in a straightforward manner from the admittance function $Y(V, \omega)$, as shown in (23)-(24).

In fact, the matrix $A(V, s)$ can be expressed in terms of a different matrix $B(V, s)$, which can be calculated both through the reduced-order formulation and at circuit level. To define the new matrix $B(V, s)$ a small-signal current source, $i_{s s}(t)$, will be introduced in parallel at the node $q$ (see Fig. 9). This source will be composed by a lower and an upper sideband about the freerunning frequency $\omega_{o}$ :

$$
i_{s s}(t)=\operatorname{Re}\left\{\Delta I_{l} e^{j\left(\omega_{o}-\Omega\right) t}+\Delta I_{u} e^{j\left(\omega_{o}+\Omega\right) t}\right\}
$$

The source will give rise to the small increments $\Delta V(t)=\operatorname{Re} \Delta V(\Omega) e^{j \Omega t}$ and $\Delta \phi(t)=\operatorname{Re} \Delta \phi(\Omega) e^{j \Omega t}$. The matrix $B(V, s)$ will relate $\Delta V(\Omega)$ and $\Delta \phi(\Omega)$ to $\Delta I_{l}, \Delta I_{u}$ through the expression:

$$
B(V, j \Omega)\left(\begin{array}{l}
\Delta V(\Omega) \\
\Delta \phi(\Omega)
\end{array}\right)=\left(\begin{array}{l}
\Delta I_{u} \\
\Delta I_{l}^{*}
\end{array}\right)
$$

To obtain the matrix $B(V, j \Omega)$, one should introduce $\Delta I_{u} e^{j \Omega t}$ on the right-hand side of (13) (after the equal sign) and $\Delta I_{l} e^{-j \Omega t}$ on the right-hand side of (19). Then, expressing $X_{ \pm 1} \approx(V+\Delta V \pm j V \Delta \phi) e^{j \phi}$ and taking (23) into account, it is straightforward to obtain:

$$
B(V, j \Omega)=\left(\begin{array}{ll}
b_{V}^{+}(V, j \Omega) & b_{\phi}^{+}(V, j \Omega)+j V Y\left(V, \omega_{o}\right) \\
b_{V}^{-}(V, j \Omega) & b_{\phi}^{-}(V, j \Omega)-j V Y\left(V, \omega_{o}\right)^{*}
\end{array}\right)
$$


where the functions $b_{V, \phi}^{ \pm}$agree with those conforming the matrix $A(V, s)$ in (24), evaluated at $s=j \Omega$.

Once the matrix $B(V, j \Omega)$ is known, the matrix $A(V, j \Omega)$ can be calculated as:

$$
A(V, j \Omega)=B(V, j \Omega)+j V\left(\begin{array}{cc}
0 & -Y\left(V, \omega_{o}\right) \\
0 & Y\left(V, \omega_{o}\right)^{*}
\end{array}\right)
$$

The matrix $B(V, j \Omega)$ can be alternatively calculated through circuit-level conversion-matrix simulations [45]-[47], using an AG with the excitation amplitude $V$, plus the small-signal current source $i_{s s}$, as shown in Fig. 1(b). The results of this numerical technique should approach those of the analytical derivation in (20) to (27). However, there will be discrepancies due to the fact that (27) relies on a derivative of the fundamental-frequency admittance function $Y$ with respect to the excitation amplitude $V$, whereas the circuit-level simulation is based on a conversion-matrix representation of the nonlinear elements.

The analysis will be illustrated through its application to the dual Van der Pol-type oscillator in Fig. 4. For the parameter values in the caption, three stable steady-state solutions coexist: a dc solution and two oscillatory solutions at the frequencies $f_{1}=907 \mathrm{MHz}$ and $f_{2}=3.9 \mathrm{MHz}$. Note that two coexisting stable steady-state solutions have disjoints basins of attractions, so the observation of one or another depends on the initial conditions. Here, the transient towards the oscillatory solution at $f_{1}=907 \mathrm{MHz}$ will be studied, setting $\omega_{o}=2 \pi f_{1}$. The steady-state amplitude is $V_{o}=2.76 \mathrm{~V}$. The considered frequency band is $\left(0, \omega_{o}=2 \pi \cdot 3.5 \mathrm{GHz}\right)$, since $f_{2}-f_{1}=3$ $\mathrm{GHz}$, and the interval in the amplitude $V$ goes from 0 to $3 \mathrm{~V}$, which should cover all the transient values. Fig. 5 shows the magnitude of $F_{V}^{+}(V, j \Omega)$, which exhibits a resonance about the frequency offset $\Omega=2 \pi \cdot 3 \mathrm{GHz}$.

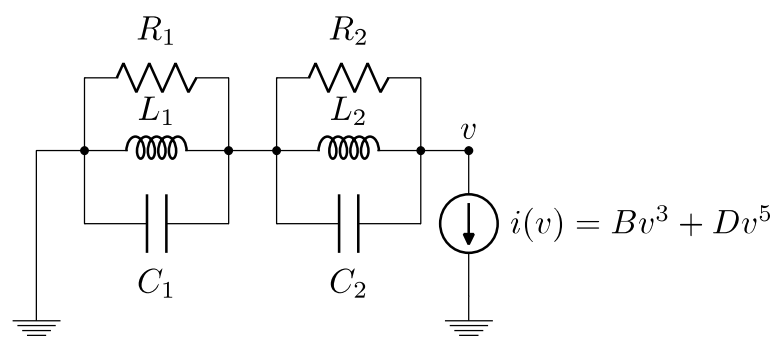

Fig. 4. Schematic of the dual Van der pol-type oscillator. The components of the resonators are $R_{1}=30 \Omega, C_{1}=1 \mathrm{nF}, R_{2}=24.44 \Omega, C_{2}=100 \mathrm{pF}$. The inductances $L_{1,2}$ are set to achieve the resonance frequencies $f_{1}=907 \mathrm{MHz}$ and $f_{2}=3.9 \mathrm{GHz}$. The respective quality factors are $Q_{1}=171$ and $Q_{2}=60$. The parameters of the nonlinear current source are $B=-0.044 \Omega^{-1} / \mathrm{V}^{2}$ and $D=0.006$ $\Omega^{-1} / \mathrm{V}^{4}$.

The application of pole-zero identification [31]-[40]] to $F_{V}^{+}(V, j \Omega)$ provides three dominant poles: $\lambda_{1}(V)=\sigma_{1}(V)$, $\lambda_{2,3}(V)=\sigma_{2}(V) \pm j \omega(V)$. The real pole $\sigma_{1}(V)$ is responsible for the growth of the amplitude of the harmonic component at the fundamental frequency $\omega_{o}$. On the other hand, the pair of complex-conjugate poles $\lambda_{2,3}(V)$ indicate the existence of an additional transient-oscillation component at $\omega_{o} \pm \omega(V)$. The real and imaginary parts of these poles are represented versus $\mathrm{V}$ in Fig. 6, where the steady-state periodic oscillation is at $V=V_{o}=2.76 \mathrm{~V}$. Between dc and this stable periodic solution, there is an unstable periodic solution of amplitude $V_{u}=1.07 \mathrm{~V}$, predicted with HB. The range of amplitude values for which the pair of complex-conjugate eigenvalues exhibit positive real part is denoted resonant range. As shown next, it will have a relevant impact on the oscillator transient response.

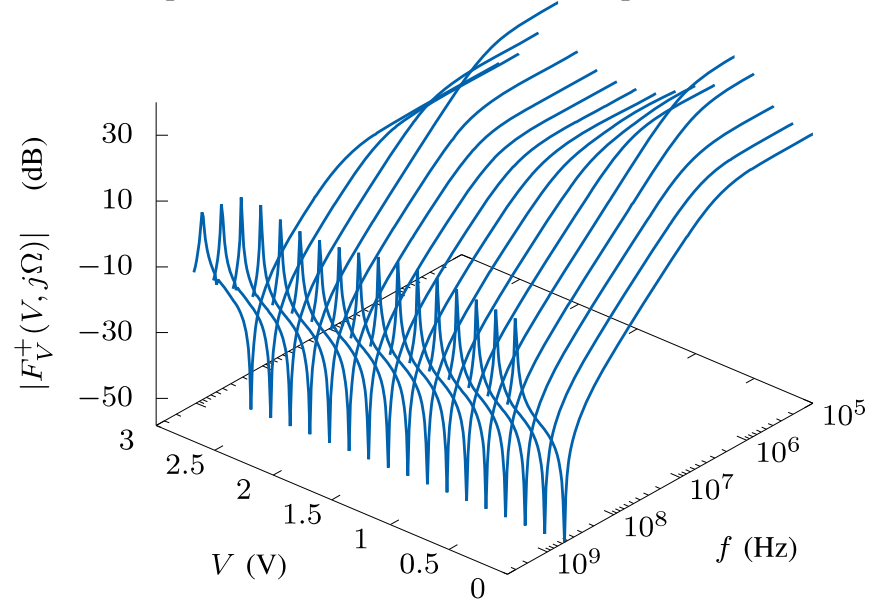

Fig. 5. Dual Van der Pol-type oscillator. Magnitude of the function $F_{V}^{+}(V, j \Omega)$ . A resonance is observed at the frequency offset $f \simeq 3 \mathrm{GHz}$, which persists for all the transient amplitude values, from dc to the steady-state solution at $V_{o}=2.76 \mathrm{~V}$.

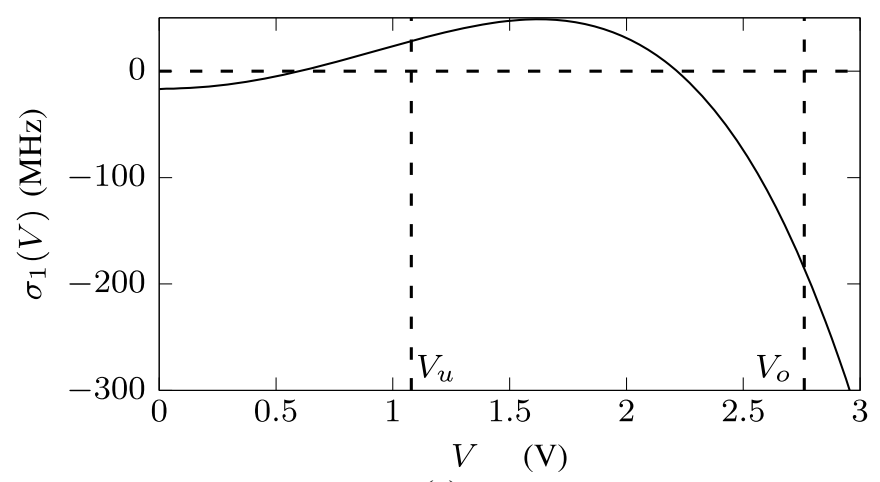

(a) 


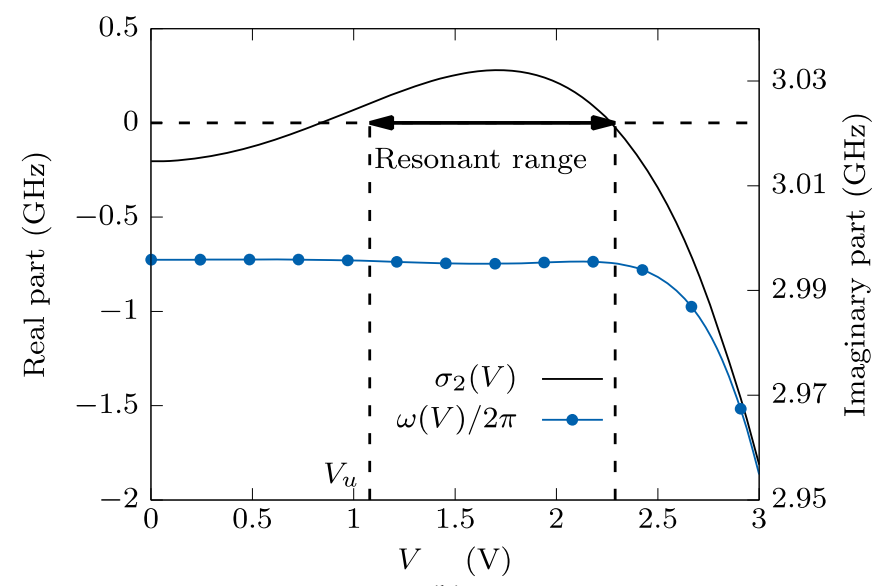

(b)

Fig. 6. Dual Van der Pol-type oscillator. Dominant generalized eigenvalues versus the first-harmonic transient amplitude $V$. (a) Real pole $\sigma_{1}(V)$. The amplitude values of the unstable $\left(V_{u}\right)$ and stable $\left(V_{o}\right)$ oscillatory solutions are indicated. (b) Real and imaginary parts of the complex-conjugate poles $\lambda_{2,3}=\sigma_{2}(V) \pm j \omega(V)$.

\section{Approximate trajectory}

Once the generalized eigenvalues are available, the goal will be to approach the transient trajectory. For the sake of clarity, the procedure will be illustrated through its application to the oscillator in Fig. 4, with $p=3$ generalized eigenvalues $\rho(V)=\left\{\sigma_{1}(V), \sigma_{2}(V)+j \omega(V), \sigma_{2}(V)-j \omega(V)\right\}$. The extension to the case $p>3$ is straightforward. At $t=t_{0}=0$, the set of initial conditions is $(V(0), \dot{V}(0), \ddot{V}(0))$, as corresponds to a third order system. Denoting $V_{0}=V(0)$, in the vicinity of the initial conditions, the amplitude variable is approached by:

$$
\begin{aligned}
& V(t) \approx V_{0}+C_{1}\left(V_{0}\right)\left(e^{\sigma_{1}\left(V_{0}\right) t}-1\right)+ \\
& C_{2}\left(V_{0}\right)\left(e^{\left[\sigma_{2}\left(V_{0}\right)+j \omega\left(V_{0}\right)\right] t}-1\right)+C_{3}\left(V_{0}\right)\left(e^{\left[\sigma_{2}\left(V_{0}\right)-j \omega\left(V_{0}\right)\right] t}-1\right)
\end{aligned}
$$

which fulfills the zero-order continuity condition $V(t=0)=V_{0}$, and the coefficients $C_{i}\left(V_{0}\right)$ are calculated by applying the continuity conditions up to the $p$-th order at $t=t_{0}=0$. In the neighborhood of the unstable solution $V_{u}$, the solution can be approached with the following linear differential equation:

$$
h_{0}\left(V-V_{0}\right)+h_{1} \dot{V}+h_{2} \ddot{V}+h_{3} \dddot{V}=0
$$

where $h_{i}$ are the coefficients of the characteristic polynomial whose roots are $\bar{\lambda}\left(V_{0}\right)$. Expressing $V_{0}=V_{u}+\varepsilon$, at $t=0$ the following equation is fulfilled:

$$
\begin{aligned}
& h_{0} \varepsilon+h_{1} \dot{V}(0)+h_{2} \ddot{V}(0)+h_{3} \dddot{V}(0)=0 \\
& \rightarrow \dddot{V}(0)=-\frac{1}{h_{3}}\left[h_{0} \varepsilon+h_{1} \dot{V}(0)+h_{2} \ddot{V}(0)\right]
\end{aligned}
$$

Then, the coefficients $C_{i}\left(V_{0}\right)$ can be obtained, in a straightforward manner, from:

$$
\begin{aligned}
& \tilde{V}_{0}=\Lambda\left(V_{0}\right) \bar{C}\left(V_{0}\right) \rightarrow \bar{C}\left(V_{0}\right)=\Lambda\left(V_{0}\right)^{-1} \tilde{V}_{0} \\
& \tilde{V}_{0} \equiv\left(\begin{array}{l}
\dot{V}(0) \\
\ddot{V}(0) \\
\dddot{V}(0)
\end{array}\right), \Lambda\left(V_{0}\right) \equiv\left(\begin{array}{ccc}
\sigma_{1}\left(V_{0}\right) & \lambda_{2}\left(V_{0}\right) & \lambda_{3}\left(V_{0}\right) \\
\sigma_{1}\left(V_{0}\right)^{2} & \lambda_{2}\left(V_{0}\right)^{2} & \lambda_{3}\left(V_{0}\right)^{2} \\
\sigma_{1}\left(V_{0}\right)^{3} & \lambda_{2}\left(V_{0}\right)^{3} & \lambda_{3}\left(V_{0}\right)^{3}
\end{array}\right), \\
& \bar{C}\left(V_{0}\right) \equiv\left(\begin{array}{l}
C_{1}\left(V_{0}\right) \\
C_{2}\left(V_{0}\right) \\
C_{3}\left(V_{0}\right)
\end{array}\right)
\end{aligned}
$$

The time interval $\Delta t_{1}=t_{1}-t_{0}$ for which (29) is assumed to be valid is determined by imposing an upper boundary to the increment undergone by the eigenvalue magnitudes. This is given by:

$$
\left|\lambda_{i}\left(V_{1}\right)-\lambda_{i}\left(V_{0}\right)\right|<\delta \lambda_{i}, \quad i=1, . .3
$$

where $V_{1}=V\left(\mathrm{t}_{1}\right)$ and $\delta \lambda_{i}$ is a small increment (e.g. $\left.\delta \lambda_{i}<0.1\left|\lambda_{i}\left(V_{0}\right)\right|\right)$. Once the interval $\Delta t_{1}$ has been established, the vector of derivatives $\tilde{V}_{1}$ is obtained from:

$\frac{d^{i} V_{1}}{d t^{i}}=\sigma_{1}\left(V_{0}\right)^{k} C_{1}\left(V_{0}\right) e^{\sigma_{1}\left(V_{0}\right) \Delta t_{1}}+\lambda_{2}\left(V_{0}\right)^{k} C_{2}\left(V_{0}\right) e^{\lambda_{2}\left(V_{0}\right) \Delta t_{1}}+$

$\lambda_{3}\left(V_{0}\right)^{k} C_{3}\left(V_{0}\right) e^{\lambda_{3}\left(V_{0}\right) \Delta t_{1}}, \quad \mathrm{i}=1,2,3$

These derivatives are then used to calculate the coefficients of the next interval, through $\bar{C}\left(V_{1}\right)=\Lambda\left(V_{1}\right)^{-1} \tilde{V}_{1}$. This recursive procedure is applied to obtain the complete trajectory, using:

$$
\begin{aligned}
& V_{k+1} \approx V_{k}+C_{1}\left(V_{k}\right)\left(e^{\sigma_{1}\left(V_{k}\right) t}-1\right)+ \\
& C_{2}\left(V_{k}\right)\left(e^{\left[\sigma_{2}\left(V_{k}\right)+j \omega\left(V_{k}\right)\right] \Delta t_{k+1}}-1\right)+C_{3}\left(V_{k}\right)\left(e^{\left[\sigma_{2}\left(V_{k}\right)-j \omega\left(V_{k}\right)\right] \Delta t_{k+1}}-1\right)
\end{aligned}
$$

In order to fulfill condition $\left|\lambda_{i}\left(V_{k+1}\right)-\lambda_{i}\left(V_{k}\right)\right|<\delta \lambda_{i}$ at each interval, it is convenient to use a variable time step $\Delta t_{k}$. However, for simplicity, in this example, the criterion $\Delta t_{k}=2 \pi / \omega\left(V_{k}\right) \approx 0.3 \mathrm{~ns}$ has been chosen, which as detailed in the following fulfils $\left|\lambda_{i}\left(V_{k+1}\right)-\lambda_{i}\left(V_{k}\right)\right|<\delta \lambda_{i}$. According to Fig. 6(a) and 6(b), the fastest amplitude variation corresponds to $V_{F} \approx 1.72 \mathrm{~V}$, for which $\sigma_{2}\left(V_{F}\right) \approx 278 \cdot 10^{6} \mathrm{~s}^{-1}$. Then, the time step is chosen so that, in the subinterval of fastest variation, the term $1-e^{\sigma_{2}\left(V_{F}\right) \Delta t}<0.1$, which keeps the growth of the fastest term in expression (35) for each interval under $10 \%$. Verifying that $\Delta t_{k}=2 \pi / \omega\left(V_{k}\right)$ fulfills this condition is straightforward. The order of $\Delta t_{k}$ is similar to the time step used in the circuitlevel envelope-transient simulation presented later in this section.

The variation of the amplitude $V$ through the transient trajectory, calculated from (35), has been represented in Fig. 7(a), as well as the magnitude of the term $U_{\Delta f}\left(t_{k}\right)=C_{2}\left(V_{k}\right) e^{\left[\sigma_{2}\left(V_{k}\right)+j \omega\left(V_{k}\right)\right] \Delta t_{k+1}}$, which gives rise to an 
additional component at $f_{1}+\Delta f\left(V_{k}\right) \approx f_{2}$. In the resonant range of Fig. 6(b), $U_{\Delta f}$ grows exponentially due to the positive value of the real part of the eigenvalues for that $V$ interval. See the effect in Fig. 7(a), where the resonant range is indicated as R. R. Note that the initial value $V$ has been set inside R. R. Beyond the resonant range, the real part of the complex-conjugate eigenvalues is negative, making this component decay. One interesting property of the LL method is that the effect of the generalized eigenvalues is fully determined by the amplitude $V$. It does not depend on the time value at which this amplitude is reached.

The resonance effect detected in (35) has been validated with circuit-level envelope transient simulations using a two-tone basis, at $f_{1}$ and $f_{2}$. One must emphasize that, using a one-tone basis at $f_{1}$, this circuit-level simulation is unable to capture this resonance, as will be shown in the next section. This is due to the large frequency difference $\Delta f$. The magnitude of the components at $f_{1}$ and $f_{2}$ is traced versus time in Fig. 7(a). As can be seen, they exhibit a good agreement with the new method. In fact, once the presence of $f_{2}$ has been detected, a circuit-level envelope transient analysis under two independent fundamental frequencies $f_{1}$ and $f_{2}$ (two-tone analysis) will provide, in general, a greater accuracy. Fig. 7(b) shows the full time domain waveform obtained from the harmonic components of the envelope-transient simulation, which evidences the resonance effects at the amplitude values predicted in Fig. 6(b) and Fig. 7(a).

A relevant finding is the possibility to match the transient response from a sequence of pole-zero identifications versus the excitation amplitude $V$. As has been shown, the poles agree with the generalized eigenvalues obtained in the local linearization of the transient at the particular node amplitude $V$. From a practical viewpoint, in circuit-level $\mathrm{HB}$ analysis, this amplitude is forced with an AG. Due to the ideal bandpass filter $\delta\left(\omega-\omega_{A G}\right)$ of this $\mathrm{AG}$, its connection to the circuit has no impact on the poles obtained for each $V$.

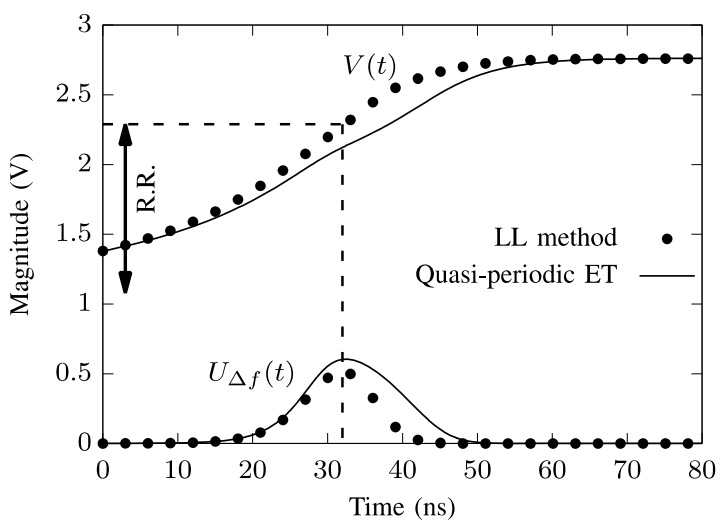

(a)

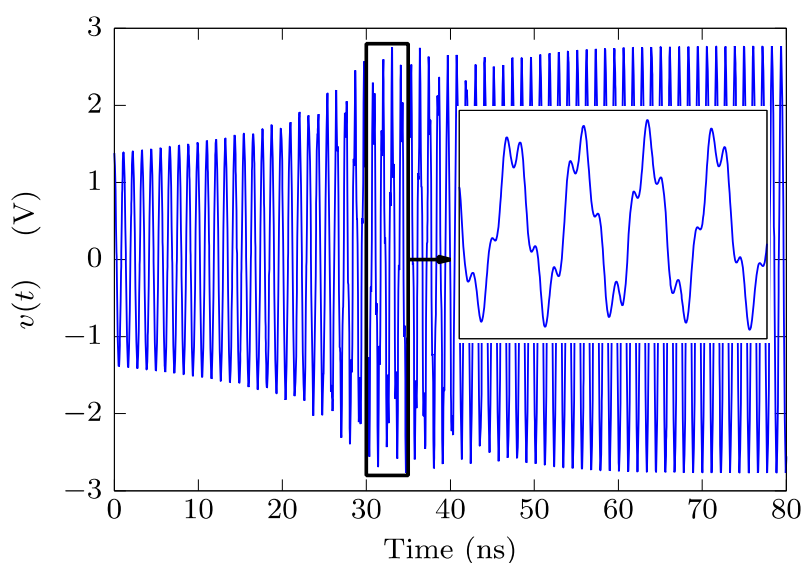

(b)

Fig. 7. Dual Van der Pol-type oscillator. (a) Time evolution of the amplitude components $U_{f_{1}}(t)$ and $U_{\Delta f}(t)$, corresponding to the frequencies $f_{1}$ and $\Delta f=f_{2}-f_{1}$, respectively. The trajectories approached by the LL method are compared with the results of a circuit-level two-tone envelope transient (ET) simulation, at the two fundamental frequencies $f_{1}$ and $f_{2}$. (b) Time-domain waveform obtained from the circuit-level envelope transient simulation.

\section{Nonlinear integration}

The nonlinear integration of equation (5), depending on a numerical reduced-order admittance function $Y(V, \omega)$, requires a previous fitting of this function. This will be carried out by sweeping the excitation amplitude $V$ and fitting, for each $V$, the real and imaginary parts of $Y(V, \omega)$ with a quotient of polynomials at each $V$ step. This fitting provides:

$$
\begin{aligned}
& Y(V, \omega)=Y\left(V, \omega_{o}+s / j\right)=\frac{c_{n}(V) s^{n}+\cdots+c_{1}(V) s+c_{0}(V)}{d_{m}(V) s^{m}+\cdots+d_{1}(V) s+d_{0}(V)}, \\
& s \equiv j\left(\omega-\omega_{o}\right)
\end{aligned}
$$

Once $Y(V, \omega)$ is represented as a quotient of polynomials, the derivation of the differential equation governing the transient is straightforward. The frequency $\omega$ is expressed as $\omega=\omega_{o}+D_{t} / j$, where $D_{t}$ is a time differentiator operator. This provides:

$$
\begin{aligned}
& Y\left(V, \omega_{o}+D_{t} / j\right) X_{1}(t)=0 \rightarrow \\
& c_{n}(V) \frac{d^{n} X_{1}}{d t^{n}}+\cdots+c_{1}(V) \frac{d X_{1}}{d t}+c_{0}(V) X_{1}=0
\end{aligned}
$$

The above equation is nonlinear in $V$ and can be solved after time discretization by applying a standard algorithm, such as the backward-Euler rule [30].

The method has been applied to the oscillator in Fig. 4. Results obtained through the integration of (37) have been superimposed in Fig. 8 and compared with circuit-level envelope transient simulation using a periodic basis at $f_{1}$. Note that none of the two methods is able to predict the resonant component, which is due to the large frequency difference $\Delta f$. The same nonlinear integration has been tested in the FETbased oscillator of Fig. 2(a). Again, as shown in Fig. 3(a) and Fig. 3(c), the results exhibit a very good agreement with those provided by circuit-level envelope-transient simulations. 


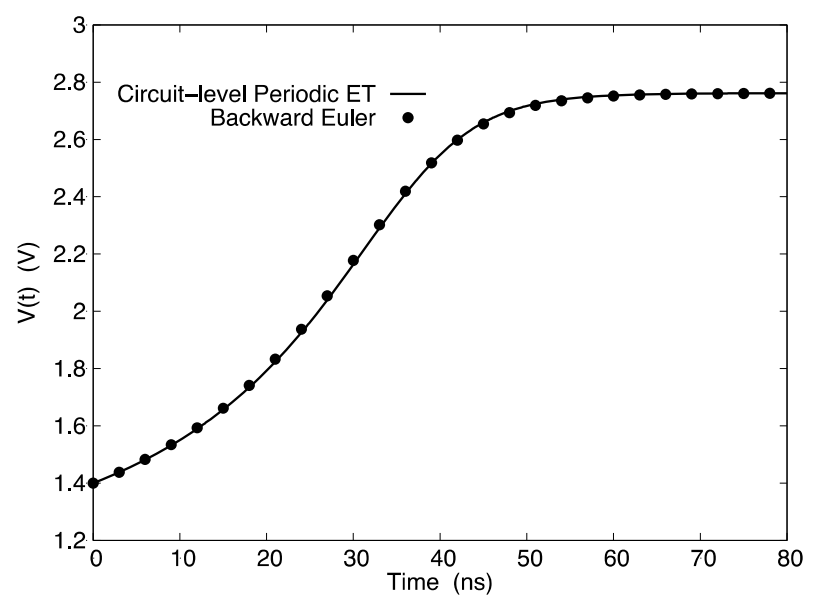

Fig. 8. Dual Van der Pol oscillator. Comparison between circuit-level envelope transient simulation using a periodic basis and backward Euler integration of equation (37)

\section{VALIDATION WITH EXPERIMENTAL MEASUREMENTS}

In the following, the transient response of several oscillators, with qualitatively different behavior, will be analyzed. The aim is to evaluate of the generality of the method through its application to a variety of circuits. Experimental measurements will be used to validate the analysis results in the case of the practical FET-based oscillators.

\section{A. Oscillator based on two cross-coupled FET transistors}

The transient of the oscillator in Fig. 9, based on two crosscoupled FET transistors [48] has been analyzed. The observation node $q$ has been placed at the coupling network. The free-running steady-state oscillation is given by $V_{o}=1 \mathrm{~V}$ and $f_{o}=2.5 \mathrm{GHz}$. The function $F_{V}^{+}(V, j \Omega)$ has been analyzed, and its magnitude is represented in Fig. 10(a), where a resonance at a frequency offset $f \simeq 700 \mathrm{MHz}$ can be noted. As can be seen in this figure, the effect of the resonance is more noticeable near the steady-state amplitude at $V_{o}=1 \mathrm{~V}$.

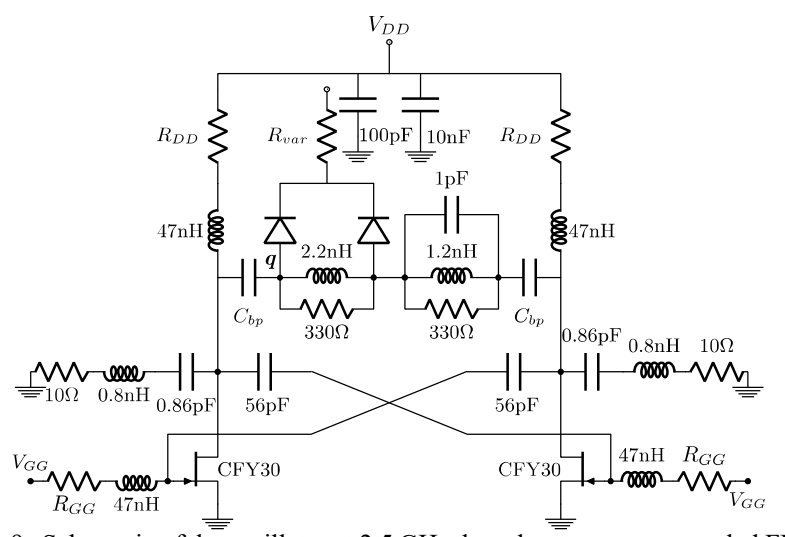

Fig. 9. Schematic of the oscillator at $2.5 \mathrm{GHz}$, based on two cross-coupled FET transistors. The prototype is implemented on RO4003C substrate $\left(\epsilon_{r}=3.35, h=32 \mathrm{mil}\right)$.

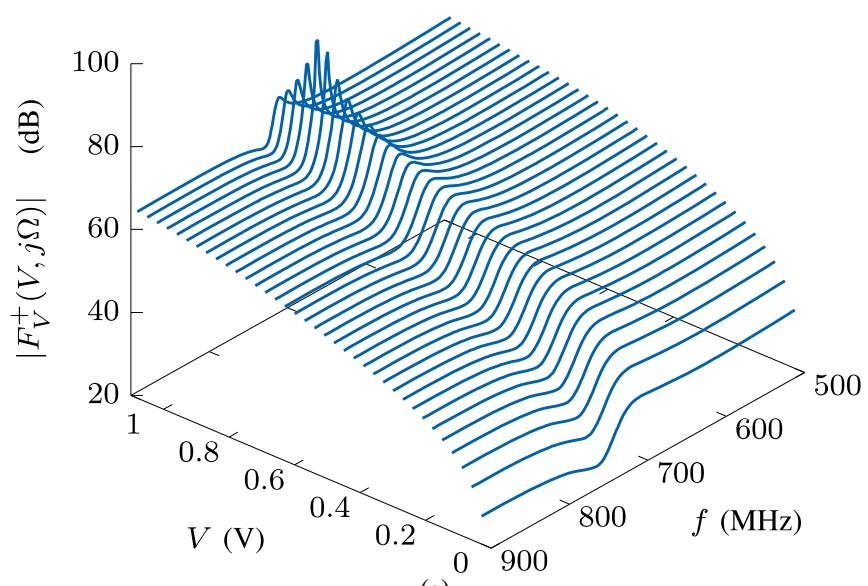

(a)

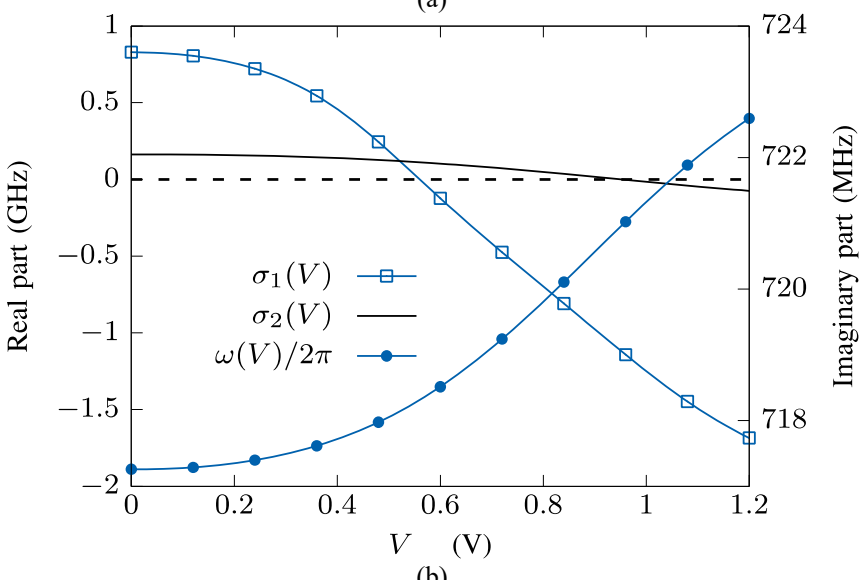

(b)

Fig. 10. Transient stability analysis of the oscillator based on two cross-coupled FET transistors. (a) Magnitude of the function $\left|F_{V}^{+}(V, j \Omega)\right|$, showing a resonance at $f \approx 720 \mathrm{MHz}$. (b) Generalized eigenvalues. The pole-zero identification of the function $F_{V}^{+}(V, j \Omega)$ determines the presence of a dominant pair of complex-conjugate poles that yield an additional frequency component during the transient, whose value is given by the imaginary part of these poles $\omega(V) / 2 \pi \approx 720 \mathrm{MHz}$.

Pole-zero identification has been applied to the function $F_{V}^{+}(V, j \Omega)$, and the dominant generalized eigenvalues are shown in Fig. 10(b). As in the previous case, there is a real pole $\sigma_{1}(V)$ governing the amplitude transient trajectory and a pair of complex-conjugate poles $\lambda_{2,3}(V)=\sigma_{2}(V) \pm j \omega(V)$, yielding an additional frequency component at $\omega / 2 \pi \simeq 700 \mathrm{MHz}$.

This result has been validated through an experimental characterization. In the measurement setup, one of the oscillator outputs is connected to a spectrum analyzer, for monitoring purposes, while the second output is connected to a Keysight Infiniium 90804A oscilloscope. The ON/OFF signal applied at the $V_{D D}$ port of the oscillator corresponds to a square signal at $f_{s q}=1 \mathrm{MHz}$ (duty cycle 50\%). In the experimental tests, frequencies in the range $1 \mathrm{kHz}$ to $1 \mathrm{MHz}$ were used with no significant changes in the results. This signal was generated by a TG1010A DDS function generator. In a previous step, the peak-to-peak voltage, and the dc offset, of the square signal are adjusted to suitable values in order to ensure a proper ON/OFF cycle. The AUX port of the function generator is connected to port- 2 of the oscilloscope and this signal is used as a trigger for 
the measurement. The measured transient is shown in Fig. 11. The output signal $v_{\text {out }}(t)$ is extracted from the transistor drain through a directional coupler, so its steady-state amplitude value differs from the one at the observation node $q$. As can be observed, the transient exhibits an additional frequency component at $f=1 / T_{a} \simeq 800 \mathrm{MHz}$. This component has been predicted by the dominant complex-conjugate generalized eigenvalues of the analysis in Fig. 10(b). In agreement with the resonance map of Fig. 10(a), the effect of this additional component is more noticeable near the steady state (higher difference between the waveform maxima).

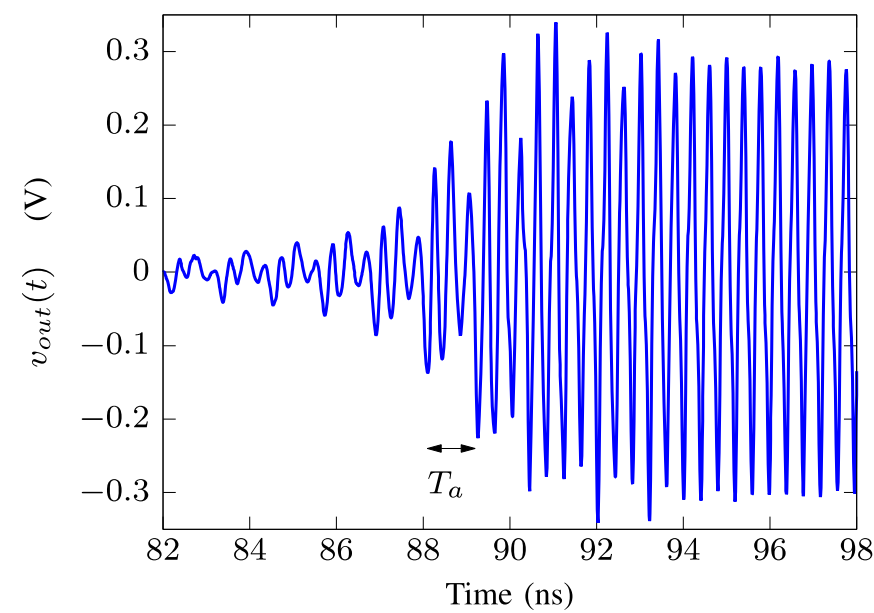

Fig. 11. Measurement of the transient state of the oscillator based on two crosscoupled FET transistors. The transient waveform of the output signal $v_{\text {out }}(t)$ has been measured with a Keysight Infiniium 90804A oscilloscope, and it shows an additional frequency component at $f=1 / T_{a} \simeq 800 \mathrm{MHz}$. As predicted by the resonance map of Fig. 10(a), the effect of this additional oscillation component is more noticeable near the steady state.

\section{B. Switched oscillator}

As shown in this subsection, the transient analysis method can also be applied to predict the response of switched oscillators. This will be illustrated through its application to the FET-based oscillator in Fig. 12(a), which is switched on and off with the varactor-bias voltage $V_{d}$. Note that it is not possible to simulate this particular oscillator with circuit-level envelope transient, even with the aid of an auxiliary generator to set the initial condition. The first stage of the analysis is a HB simulation of the oscillator circuit versus the control voltage. The results are shown in Fig. 12(b), where the first harmonic amplitude $V_{o}$ and the free-running oscillation frequency $f_{o}$ have been traced versus $V_{d}$. As can be seen, as $V_{d}$ increases the oscillation amplitude decreases, eventually becoming zero at a Hopf-type bifurcation point [42]. Beyond this point, the circuit does not oscillate.

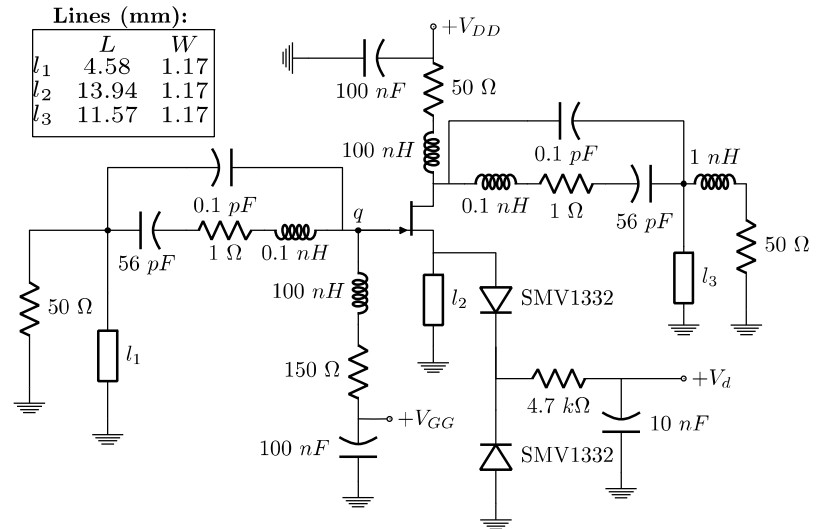

(a)

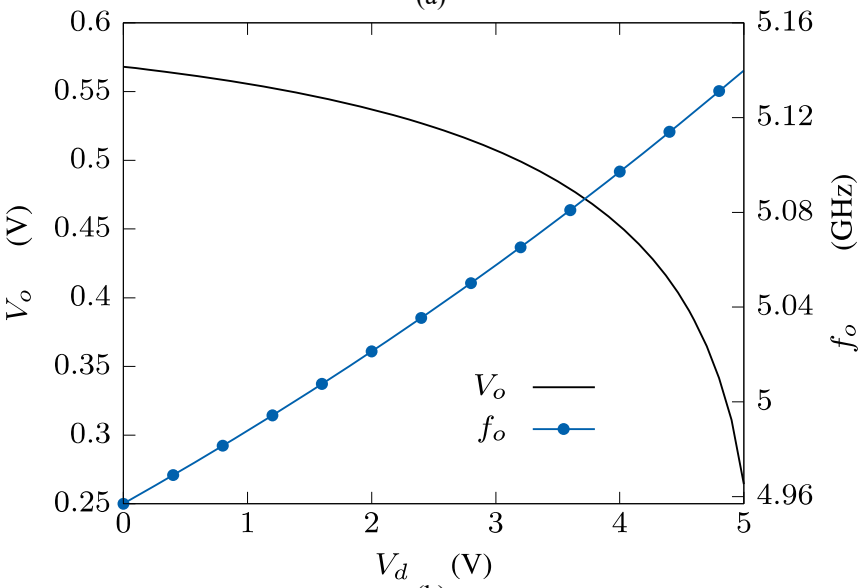

(b)

Fig. 12. Switched oscillator. (a) Schematic of the FET-based oscillator, where the variables $L$ and $W$ represent the length and width of the circuit transmission lines. The prototype is implemented on RO4003C substrate $\left(\epsilon_{r}=3.35, h=32 \mathrm{mil}\right)$ (b) First harmonic amplitude $V_{o}$ and free-running frequency $f_{o}$ of the oscillatory solution traced versus the varactor-bias voltage $V_{d}$. They have been calculated with circuit-level HB.

Now, the $V_{d}(t)$ signal will be used to switch the oscillator on and off. In fact, to perform a realistic simulation, $V_{d}(t)$ will be given by a low-frequency square signal extracted from the measurement. This way, the non-idealities of this signal will be taken into account in the simulation. The evaluation of the function $\left|F_{V}^{+}(V, j \Omega)\right|$ indicates that the oscillator transient is governed by a single real pole. The growth-rate function is calculated for each $V_{d}$ value in the rectangular signal excursion, obtaining the bivariate function $g\left(V, V_{d}\right)$ represented in Fig. 13(a). For each $V_{d}$, the functions $a_{0}\left(V, V_{d}\right)$ and $a_{1}\left(V, V_{d}\right)$ in (7) are evaluated at the free-running frequency $\omega_{o}\left(V_{d}\right)$, obtained from the HB simulation of Fig. 12(b).

In Fig. 13(a), the oscillator response to the time-varying signal $V_{d}(t)$ has been represented in the phase-space $\left(\dot{V}, V, V_{d}\right)$. The trajectory has been calculated by numerically solving the nonautonomous ODE:

$$
\dot{V}=g\left(V, V_{d}(t)\right)
$$


All system trajectories lie in the surface determined by the bivariate function $g\left(V, V_{d}\right)$. In Fig. 13(b), the first harmonic amplitude $V(t)$ resulting from the ODE (38) is compared with the measurement in a Keysight Infiniium 90804A oscilloscope. The measured time-varying voltage $V_{d}(t)$ is superimposed in the same figure. As can be seen in Fig. 13(b), the ODE (38) predicts accurately the asymptotic response of the VCO to a step-shaped modulation signal. For this reason, the proposed ODE can be a good alternative in oscillator circuits where the envelope-transient technique is unable to converge.

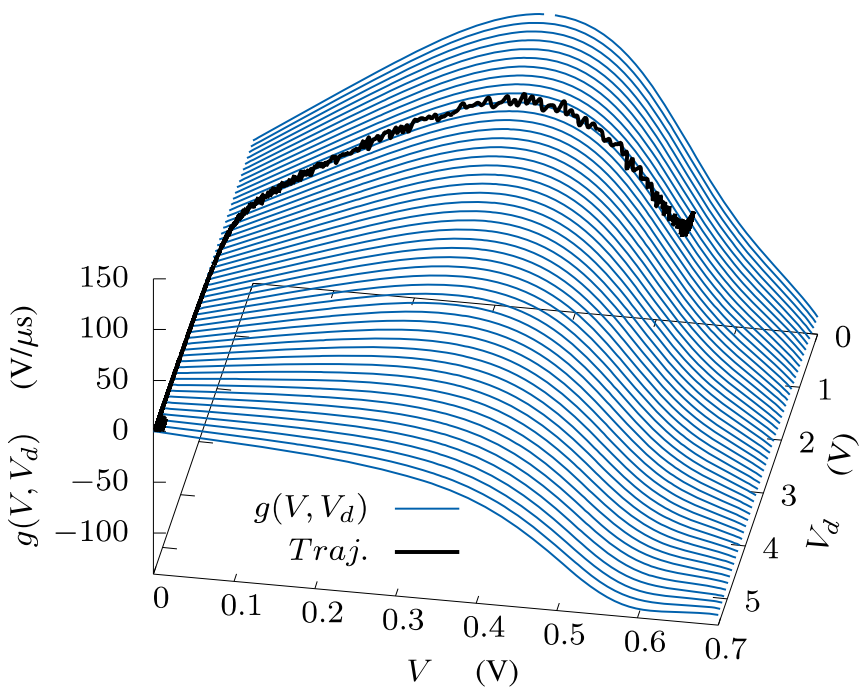

(a)

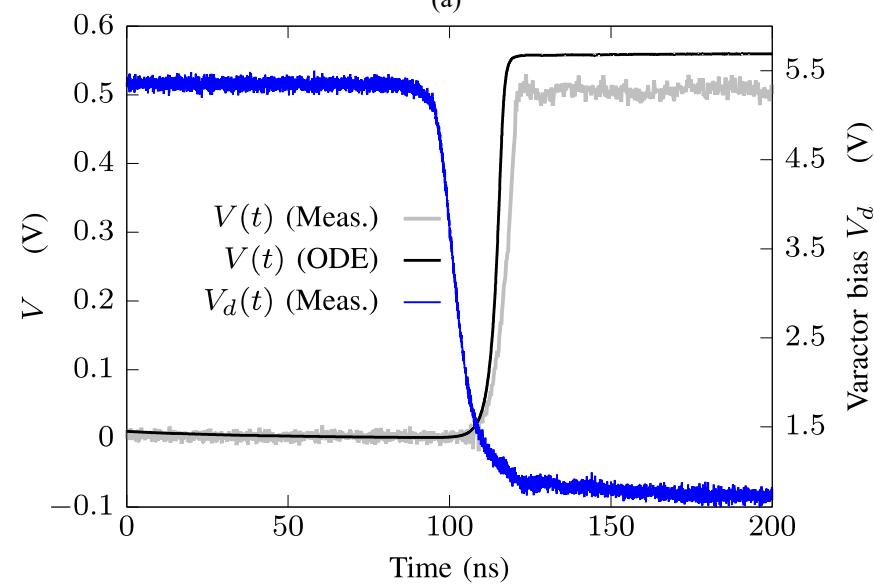

(b)

Fig. 13. Switched oscillator. (a) Bivariate function $g\left(V, V_{f}\right)$. All the

trajectories in the phase space $\left(\dot{V}, V, V_{d}\right)$ lie in the surface determined by this function. (b) Oscillator response to a modulated bias voltage $V_{d}(t)$. The time evolution of the first harmonic amplitude $V(t)$ is calculated from the ODE (38) and compared with the measurement in a Keysight Infiniium 90804A oscilloscope. The measured time-varying voltage $V_{d}(t)$ is superimposed on the same figure. The signal $V_{d}(t)$ has been imported from the experimental source TG1010A DDS.

\section{CONCLUSION}

A new method for the understanding of the mechanisms that govern the oscillator transient dynamics has been presented. It is based on the application of the implicit-function theorem to the harmonic-balance system to obtain a nonlinear admittance function from a given observation node. The resulting reducedorder envelope-domain equation can be solved with a locallinearization method or, more accurately, with a nonlinear iterative technique. In the first case, a sequence of linear ordinary differential equations is derived, which depend on the excitation amplitude and describe the system dynamics in the vicinity of each point of the transient amplitude trajectory. Departing from this sequence of equations, a set of generalized eigenvalues is calculated, which govern the acceleration or deceleration of the oscillation growth and are responsible for the onset and extinction of spurious oscillations. As an additional contribution, the concept of escape time, or the time required by the transient trajectory to go through a given interval of amplitude values, has also been introduced. Various applications of the new model have been presented, including a dual-frequency oscillator and a switched oscillator. The results in all the practical FET-based oscillator have been successfully compared with experimental measurements.

\section{ACKNOWLEDGEMENT}

This work was supported by the Spanish Ministry of Economy and Competitiveness and the European Regional Development Fund (ERDF/FEDER) under Project TEC2014-60283-C3(1/2)-R and Project TEC2017-88242-C3-(1/2)-R. The authors are grateful to AMCAD Engineering for technical support.

\section{REFERENCES}

[1] P. Gamand and V. Pauker, "Starting phenomenon in negative resistance FET oscillators," Electron. Lett., vol. 24, no. 15, pp. 911913, Jul. 1988.

[2] B. Voigt, D. Seefeldt, and E. H. Horneber, "A new approach to determine the start-up time of sinusoidal oscillators," 37th Midwest Symp. Circ. Syst., 1994, vol. 2, pp. 1119-1122.

[3] Y. Tsuzuki, T. Adachi, and Ji Wen Zhang, "Formulation of nonlinear negative resistance for calculation of start-up characteristics of crystal oscillators," IEEE Int. Freq. Control Symp., 1996, pp. 710-713.

[4] H. Meyr and Ascheid, G., Synchronization in Digital Communications, vol. 1. New York: Wiley Interscience, 1990.

T. H. Lee and A. Hajimiri, "Oscillator phase noise: a tutorial," IEEE J. Solid-State Circuits, vol. 35, no. 3, pp. 326-336, Mar., 2000.

[6] R. D. Hempstead and M. Lax, "Classical Noise. VI. Noise in SelfSustained Oscillators near Threshold," Phys. Rev., vol. 161, no. 2, pp. 350-366, Sep. 1967.

[7] M. Lax, "Classical Noise. V. Noise in Self-Sustained Oscillators," Phys. Rev., vol. 160, no. 2, pp. 290-307, Aug. 1967.

[8] E. Ngoya, J. Rousset, and D. Argollo, "Rigorous RF and Microwave Oscillator Phase Noise Calculation by Envelope Transient Technique," in IEEE MTT-S Symposium, 2000, pp. 91-94.

[9] K. Kundert, "Introduction to RF simulation and its application," in Proceedings of the 1998 IEEE Bipolar/BiCMOS Circuits and Technology Meeting, 1998, pp. 67-78.

[10] N. B. Carvalho, J. C. Pedro, and W. Jang, "Nonlinear RF circuits and systems simulation when driven by several modulated signals," IEEE Trans. Microw. Theory Tech., vol. 54, no. 2, pp. 572-579, Feb., 2006.

[11] A. Suarez, E. Fernandez, F. Ramirez, and S. Sancho, "Stability and bifurcation analysis of self-oscillating quasi-periodic regimes," IEEE Trans. Microw. Theory Tech., vol. 60, no. 3, pp. 528-541, Mar., 2012. V. Rizzoli, A. Neri, and F. Mastri, "A modulation-oriented piecewise harmonic-balance technique suitable for transient analysis and digitally modulated signals," in 1996 26th European Microwave Conference, EuMC 1996, 1996, vol. 2, pp. 546-550.

[13] A. Suárez, S. Sancho, and F. Ramírez, "General Formulation for the Analysis of Injection-Locked Coupled-Oscillator Systems," IEEE 
Trans. Microw. Theory Tech., vol. 61, no. 12, pp. 4730-4744, Dec. 2013.

[14] A. Suárez, F. Ramírez, and S. Sancho, "Stability and noise analysis of coupled-oscillator systems," IEEE Trans. Microw. Theory Tech. vol. 59, no. 4 PART 1, pp. 1032-1046, Apr. 2011.

[15] T. Heath, "Beam steering of nonlinear oscillator arrays through manipulation of coupling phases," IEEE Trans. Antennas Propag., vol. 52, no. 7, pp. 1833-1842, Jul., 2004.

[16] S. Nogi, J. Lin, and T. Itoh, "Mode analysis and stabilization of a spatial power combining array with strongly coupled oscillators," IEEE Trans. Microw. Theory Tech., vol. 41, pp. 1827-1837, Oct., 1993.

[17] R. A. York and T. Itoh, "Injection- and phase-locking techniques for beam control," IEEE Trans. Microw. Theory Tech., vol. 46, no. 11, pp. 1920-1929, Nov., 1998.

[18] R. A. York, "Nonlinear analysis of phase relationships in quasioptical oscillator arrays," IEEE Trans. Microw. Theory Tech., vol. 41, no. 10, pp. 1799-1809, Oct., 1993.

[19] A. Suarez, S. Sancho, and F. Ramirez, "Growth-rate function for the nonlinear analysis of the transient dynamics of microwave oscillators," in IEEE MTT-S International Microwave Symposium Digest, 2016, pp. 1-4.

[20] A. Suárez, M. Pontón, S. Sancho, and F. Ramírez, "New methodologies for the analysis and synthesis of oscillator circuits," in 2018 IEEE MTT-S Latin America Microwave Conference, LAMC 2018 - Proceedings, 2019, pp. 1-3.

[21] K. Kurokawa, "Some basic characteristics of broadband negative resistance oscillator circuits," Bell Syst. Tech. J., vol. 48, no. 6, pp. 1937-1955, Jul. 1969.

[22] J. Ramos, "Linearized methods for ordinary differential equations.," Appl. Math. Comput., vol. 104, pp. 109-129, Sep., 1999.

[23] J. C. Jimenez and F. Carnodell, "Rate of convergence of local linearization schemes for initial-value problems," Appl. Math. Comput., vol. 171, pp. 1282-1295, Dec., 2005.

[24] J. C. Jimenez, R. Biscay, C. Mora, and L. M. Rodriguez, "Dynamic properties of the local linearization method for initial-value problems," Appl. Math. Comput., vol. 126, no. 1, pp. 63-81, Feb., 2002.

[25] G. Cook and C. F. Lin., "Comparison of a local linearization algorithm with standard numerical integration methods for real-time simulation," IEEE Trans. Ind. Electron. Control Instrum., vol. 27, no. 3, pp. 129-132, Aug., 1980.

[26] G. Gil, R. G. Sanfelice, and P. E. Nikravesh, "Local Linearization Method in the Integration of Multibody Equations," in 2013 ECCOMAS Thematic Conference on Multibody Dynamics, 2013, pp. 613-622.

[27] L. Petzold, S. Li, Y. Cao, and R. Serban, "Sensitivity analysis of differential-algebraic equations and partial differential equations," Comput. Chem. Eng., vol. 30, no. 10-12, pp. 1553-1559, Sep., 2006. R. P. Dickinson and R. J. Gelinas, "Sensitivity Analysis of Ordinary Differential Direct Method," J. Comput. Phys., vol. 143, no. 21, pp. 123-143, Jun., 1976.

[29] B. R. E. Scheid, "The Accurate Numerical Solution of Highly Oscillatory Ordinary Differential Equations," Acta Numer., vol. 41, no. 164 , pp. $487-509$, Oct., 1983.

[30] T. S. Parker, and L. O. Chua, Practical Numerical Algorithms for Chaotic Systems., vol. 56, no. 193. Springer-Verlag, 2006.

[31] J. Jugo, A. Anakabe, and J. M. Collantes, "Control design in the harmonic domain for microwave and RF circuits," IEE Proc. Control Theory Appl., vol. 150, no. 2, pp. 127-131, Mar. 2003.

[32] J. Jugo, J. Portilla, A. Anakabe, A. Suarez, and J. M. Collantes, "Closed-loop stability analysis of microwave amplifiers," IEE Electron. Lett., vol. 37, pp. 226-228, Feb., 2001.

[33] A. Anakabe et al., "Analysis and elimination of parametric oscillations in monolithic power amplifiers," in IEEE MTT Symposium, 2002, pp. 2181-2184.

[34] A. Anakabe, N. Ayllón, J. M. Collantes, A. Mallet, G. SoubercazePun, and K. Narendra, "Automatic pole-zero identification for multivariable large-signal stability analysis of RF and microwave circuits," in Microwave Conference (EuMC), 2010 European, 2010, no. September, pp. 477-480.

[35] N. Ayllón, A. Anakabe, J. M. Collantes, G. Soubercaze-Pun, and S. Forestier, "Sensitivity enhancement in pole-zero identification based stability analysis of microwave circuits," in INMMIC 2008 Workshop on Integrated Nonlinear Microwave and Milimetre-Wave
Circuits, Proceedings, 2008, pp. 75-78.

[36] N. Ayllon, J. Collantes, A. Anakabe, I. Lizarraga, G. SoubercazePun, and S. Forestier, "Systematic Approach to the Stabilization of Multitransistor Circuits," IEEE Trans. Microw. Theory Tech., vol. 59, no. 8, pp. 2073-2082, Aug., 2011.

[37] N. Ayllon, J.-M. Collantes, A. Anakabe, G. Soubercaze-Pun, S. Forestier, and D. Langrez, "Joint RF and large-signal stability optimization of MMIC power combining amplifiers," Int. J. Microw. Wirel. Technol., vol. 5, no. 6, pp. 683-688, Dec., 2013.

[38] N. Otegi, A. Anakabe, J. Pelaz, J. Collantes, and G. Soubercaze-Pun, "Experimental Characterization of Stability Margins in Microwave Amplifiers," IEEE Trans. Microw. Theory Tech., vol. 60, no. 12, pp. 4145-4156, Dec., 2012

[39] J. Pelaz, J. Collantes, N. Otegi, A. Anakabe, and G. Collins, "Experimental Control and Design of Low-Frequency Bias Networks for Dynamically Biased Amplifiers," IEEE Trans. Microw. Theory Tech., vol. 63, no. 6, pp. 1923-1936, Jun., 2015.

[40] L. Mori et al., "Stability analysis of multistage power amplifiers using Multiple-Input Multiple-Output identification," in 2016 IEEE MTT-S International Microwave Symposium (IMS), 2016, pp. 1-4.

[41] S. Wiggins, Introduction to Applied Nonlinear Dynamical Systems and Chaos. New York: Springer-Verlag, 1990.

[42] A. Suarez, Analysis and Design of Autonomous Microwave Circuits. Wiley, 2009.

[43] A. Suárez and S. Sancho, "Application of the envelope-transient method to the analysis and design of autonomous circuits," Int. J. RF Microw. Comput. Eng., vol. 15, no. 6, pp. 523-535, Nov., 2005.

[44] D. H. Trahan, W. E. Boyce, and R. C. DiPrima, Elementary Differential Equations and Boundary Value Problems., vol. 86, no. 7. John Wiley \& Sons, 2006.

[45] J. M. Paillot, J. C. Nallatamby, M. Hessane, R. Quere, M. Prigent, and J. Rousset, "A general program for steady state, stability, and FM noise analysis of microwave oscillators," in IEEE MTT-S International Microwave Symposium Digest, 2002, pp. 1287-1290.

[46] P. Bolcato, J. C. Nallatamby, R. Larcheveque, M. Prigent, and J. Obregon, "A unified approach of PM noise calculation in large RF multitone autonomous circuits," in IEEE MTT Symposium, 2000, pp. $417-420$.

[47] S. A. Maas, Nonlinear Microwave Circuits. Norword, Ma: Artech House, 1997.

[48] F. Ramírez, S. Sancho, and A. Suárez, "Oscillation Modes in Multiresonant Oscillator Circuits," IEEE Trans. Microw. Theory Tech., vol. 64, no. 12, pp. 4660-4675, Dec., 2016.

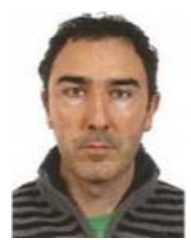

Sergio Sancho (A'04-M'04) received the degree in Physics from Basque Country University in 1997. In 1998 he joined the Communications Engineering Department of the University of Cantabria, Spain, where he received the Ph.D. degree in Electronic Engineering in February 2002. At present, he works at the University of Cantabria, as an Associate Professor of its Communications Engineering Department. His research interests include the nonlinear analysis of microwave autonomous circuits and frequency synthesizers, including stochastic and phase-noise analysis.

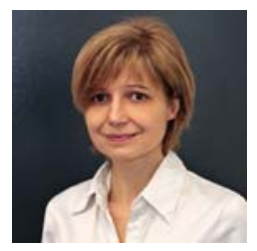

Almudena Suárez (M'96-SM'01-F'12) was born in Santander, Spain. She received the Electronic Physics and Ph.D. degrees from the University of Cantabria, Santander, Spain, in 1987 and 1992, respectively, and the Ph.D. degree in Electronics from the University of Limoges, Limoges, France, in 1993.

She is currently a Full Professor with the Communications Engineering Department, University of Cantabria. She co-authored Stability Analysis of Nonlinear Microwave Circuits (Artech House, 2003) and authored Analysis and Design of Autonomous Microwave Circuits (IEEE-Wiley, 2009).

Prof. Suárez is a member of the Technical Committees of the IEEE Microwave Theory and Techniques Society (IEEE MTT-S) International Microwave Symposium (IMS) and the European Microwave Conference. She 
was an IEEE Distinguished Microwave Lecturer from 2006 to 2008. She is a member of the Board of Directors of the European Microwave Association. She was the Coordinator of the Communications and Electronic Technology Area for the Spanish National Evaluation and Foresight Agency between 2009 and 2013. In 2014 and 2015 she was the co-chair of IEEE Topical Conference on RF Power Amplifiers (PAWR). Prof. Suárez is the Editor-in-Chief of the International Journal of Microwave and Wireless Technologies from Cambridge University Press journals and an Associate Editor for IEEE Microwave Magazine.

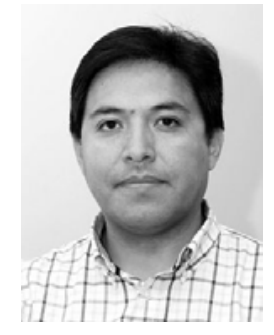

Franco Ramírez (S'03-A'05-M'05-SM'16) obtained a degree in electronic systems engineering degree from the Military School of Engineering (EMI) in La Paz, Bolivia, in 2000 and the Ph.D. degree in Communications Engineering from the University of Cantabria, Santander, Spain in 2005. From 1999 to 2000, he worked for Ericsson de Bolivia Telecomunicaciones, where he was involved in projects related with GSM and TDMA technologies. From 2009 to 2005, Dr. Ramírez was a Research Fellow of the "Ramón y Cajal" Programme, funded by the Spanish Ministry of Science and Innovation, at the Communications Engineering Department of the University of Cantabria, where he is currently an Associate Professor. His research interests include phase noise, stability and the development of nonlinear techniques for the analysis and design of autonomous microwave circuits.

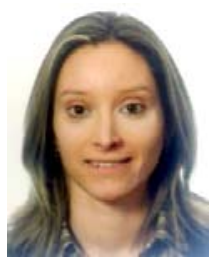

Mabel Pontón (S'08-M'11) was born in Santander, Spain. She received the bachelor's degree in Telecommunication Engineering, master's degree in Information Technologies and Wireless Communications Systems, and Ph.D. degree from the University of Cantabria, Santander, in 2004, 2008, and 2010, respectively. In 2006, she joined the Communications Engineering Department, University of Cantabria. From 2011 to 2013, she was with the Group of Electronic Design and Applications, Georgia Institute of Technology, Atlanta, GA, USA, as a Post-Doctoral Research Fellow.

Her current research interests include the nonlinear analysis and simulation of radiofrequency and microwave circuits, with an emphasis on phase-noise, stability, and bifurcation analysis of complex oscillator topologies. 